\title{
Bismuth coordination polymers: from centuries-old medicines to unprecedented topological complexity
}

\author{
Andrew Kentaro Inge ${ }^{1}$, Yunchen Wang ${ }^{1}$, Sofia Takki ${ }^{1}$, Ocean Cheung ${ }^{2}$, Hongyi $\mathrm{Xu}^{1}{ }^{1}$, Wei Wan ${ }^{1}$, Lars Öhrström ${ }^{3}$, Xiaodong Zou $^{1}$, \\ Michael O'Keeffe ${ }^{4}$, Norbert Stock ${ }^{5}$ \\ ${ }^{1}$ Stockholm University, Stockholm, Sweden, ${ }^{2}$ Uppsala University, Uppsala, Sweden, ${ }^{3}$ Chalmers University of Technology, \\ Gothenburg, Sweden, ${ }^{4}$ Arizona State University, Phoenix, United States, ${ }^{5}$ University of Kiel, Kiel, Germany \\ E-mail: andrew.inge@mmk.su.se
}

Bismuth is a rather unusual heavy metal: it is practically the heaviest stable element, it is rather inexpensive and unlike its neighbors on the periodic table its compounds are generally nontoxic. For over three centuries bismuth salts and compounds have been used in cosmetics, pigments and as the active pharmaceutical ingredients (APIs) in medicines to treat a variety of diseases and conditions ranging from gastrointestinal issues, syphilis and wounds. However despite their long history in such applications, the structures and chemical formulas of many of these compounds remain unknown. Therefore it does not come as a surprise that the mechanisms in which these bismuth compounds function as APIs are poorly understood, preventing researchers from rationally designing improved bismuth-based drugs. Structure determination by conventional methods is often impeded by a number of complications. Single crystal X-ray diffraction is often not an option due to small crystallite size. X-ray powder diffraction can also be challenging due to severe preferred orientation effects as well as the complicated nature of the structures themselves. Electron diffraction often results in degradation or complete loss of crystallinity in organic and metal-organic samples due to beam sensitivity.

With the aid of recently developed fast electron diffraction tomography techniques[1] combined with a sample cooling stage we have recently elucidated the crystal structure of bismuth subgallate,[2] a compound that has been used in medicine for its antimicrobial, astringent, hemostatic and deodorizing properties since at least the 1800 s. It still continues to be used as

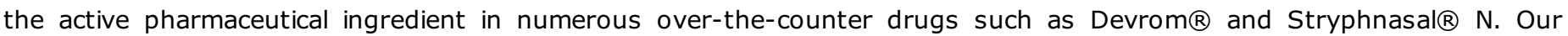
findings indicate that bismuth subgallate is actually a microporous layered coordination polymer. The chemical formula as determined from the crystal structure contradicts all previously suggested formulas as reported in the literature, chemical databases and pharmacopoeia. Elucidation of the porous structure prompted us to study gas sorption properties of the material. Bismuth subgallate has a high selectivity of adsorbing $\mathrm{CO} 2$ over N2, which may pave the way for new applications for the material. As gallic acid, the organic ligand, is naturally found in plants such as oak bark and gall nuts, the work falls within the scope of green chemistry especially since typically fossil-based organic linkers are used to synthesize coordination polymers.

We have also recently reported the crystal structure of a novel bismuth trimesate, denoted CAU-17.[3] CAU-17 is a metalorganic framework and is one of only a small number of microporous bismuth-based materials. In situ $\mathrm{X}$-ray powder diffraction studies performed within a custom-made reactor at synchrotron radiation facilities exemplify the importance of short reaction times for the synthesis of these metastable open-framework materials and the significance of crystalline intermediate phases. Topological analysis revealed that the underlying net of CAU-17 is over five times more complicated than any other MOF we are aware of and is constructed of 54 unique nodes and 135 edges. Figure is reprinted with permission from [3] Copyright 2017 American Chemical Society.

[1] Gemmi, M. et al. (2015). J. Appl. Crystallogr. 48, 718-727.

[2] Wang, Y. et al. (2017) In preparation.

[3] Inge, A. K. et al. (2016). J. Am. Chem. Soc. 138, 1970-1976.

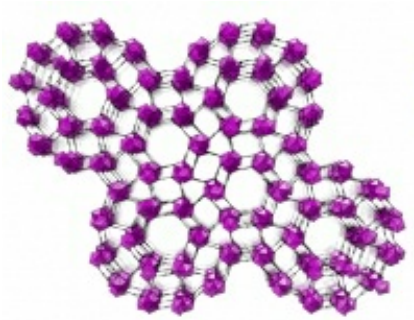

CAU-17

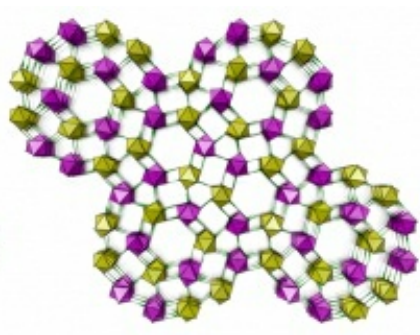

underlying net

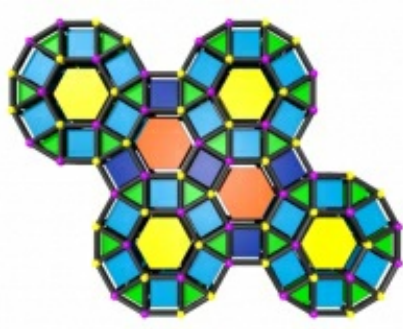

rod-packing

Keywords: bismuth, coordination polymers, structure determination 